\title{
Assessing Rice Chlorophyll Content with Vegetation Indices from Hyperspectral Data
}

\author{
Xingang Xu* ${ }^{*}$ Xiaohe Gu, Xiaoyu Song, Cunjun Li, and Wenjiang Huang \\ National Engineering Research Center for Information Technology in Agriculture, \\ P.O. Box 2449-26, Beijing 100097, P.R. China \\ xxg2007@yahoo.com.cn, guxh@nercita.org.cn, \\ songxy@nercita.org.cn, licj@nercita.org.cn, \\ huangwj@nercita.org.cn
}

\begin{abstract}
Leaf chlorophyll content is not only an important biochemical parameter for determinating the capacity of rice photosynthesis, but also a good indicator of crop stress, nutritional state. Due to the reliable, operational and nondestructive advantages, hyperspectral remote sensing plays a significant role for assessing and monitoring chlorophyll content. In the study, a few of typical vegetation indices (VI) with the combination of $670 \mathrm{~nm}$ and $800 \mathrm{~nm}$ band reflectance, Normalized Difference Vegetation Index (NDVI), Modified Simple Ratio index (MSR), Modified Chlorophyll Absorption Ratio Index (MCARI), Transformed Chlorophyll Absorption Ratio Index (TCARI), and Optimized Soil-Adjusted Vegetation Index (OSAVI) are modified by using $705 \mathrm{~nm}$ and $750 \mathrm{~nm}$ band reflectance so as to reduce the effect of spectral saturation in 660$680 \mathrm{~nm}$ absorptive band region, and then used to assess the rice chlorophyll content. The result shows that the five mentioned VIs have better correlation with rice chlorophyll content while using $705 \mathrm{~nm}$ and $750 \mathrm{~nm}$. In addition, in the study the Weight optimization combination (WOC) principle is utilized to further assess the capacity of the five modified VIs for estimating rice chlorophyll content, it is proved that OSAVI and MSR display the better performance.
\end{abstract}

Keywords: Chlorophyll Content, Vegetation Indices, Weight Optimization Combination, Hyperspectral Remote Sensing.

\section{Introduction}

Chlorophyll is one of most important matters for crop photosynthesis, and the amount of chlorophyll in crops can reflect whether the growth state of crop is in health or not. Healthy and stressed crops often display changes in pigment levels (Niinemets et al., 1997; Penuelas, J., Filella , 1998; Rasmus and Martha, 2009). In addition, chlorophyll content is firmly related with nitrogen level. Consequently, accurately assessing the chlorophyll content is very useful for obtaining crop productivity, probing crop stresses and nutritional state (Zarco-Tejada et al., 2004).

\footnotetext{
${ }^{*}$ Corresponding author.
} 
Hyperspectral remote sensing plays a unique role in detecting crop biochemical parameters, such as dry matter, pigments and so on. In the visible regions, crops present the different changes of spectrographic curve, and leaf chlorophyll content is the most important factor leading to the leaf spectral variation(Zhang et al., 2008), which provides the base for remote detection of crop growth state through monitoring chlorophyll content. Vegetation indices (VIs) with the combination of different bands can reduce some noise caused by external factors such as the atmosphere and the soil background to some extent (Demarez et al., 2000; Rasmus and Eva, 2008), and are widely used to monitor and assess the leaf chlorophyll content.

The typical VIs based on hyperspectral remote sensing, such as NDVI (Rouse et al., 1974; Gitelson et al., 1994), MSR (Chen, 1996), MCARI, TCARI (Daughtry et al., 2000), and OSAVI (Rondeaux et al., 1996), commonly use 670nm to estimate leaf chlorophyll content. However, in 660-680nm red spectral region, the chlorophyll absorptions begin to show the saturated tendency at lower chlorophyll content, which would weaken the sensitivity of VIs to higher chlorophyll content. By contrast, the absorptions in the region around $550 \mathrm{~nm}$ or $700 \mathrm{~nm}$ show the same phenomena at higher chlorophyll content (Sims and Gamon, 2002). So the spectral indices with these bands in the latter regions would have better precision for assessing chlorophyll content.

The objective of the study is to compare the performance of the above five VIs for assessing chlorophyll content by using different bands combination of reflectance to modify these indices. In addition, the Weight optimization combination (WOC) principle is utilized to further analyze the capacity of the five modified VIs for estimating rice chlorophyll content.

\section{Material and Method}

\subsection{The Study Area}

The study site is located in Qianjin and Youyi Farm, Heilongjiang Nongken, China. In the two farms, rice is main crop. Specially in Qianjin Farm, rice accounts for about $95 \%$ of total crop planting areas. In the study, there are thirty-eight paddy fields to be selected to obtain the spectral and chlorophyll content data, and these selected paddy fields are homogeneous for rice breed and field management. The collections of data are carried out on August 6, 2009.

\subsection{Field Data Acquisition}

In the paper, canopy spectral data in every selected paddy field are collected at 380 $2500 \mathrm{~nm}$ by a portable spectroradiometer (FS-FR2500, ASD, USA) with field of view of $25^{\circ}$ and a distance of about $150 \mathrm{~cm}$ above the ground surface. Reflectance spectra are derived through calibration with the $99 \%$ white reference board (Labsphere, Inc., North Sutton, New Hampshire, USA).The measured spectra data for ten times are averaged as the final spectral data in every paddy field.

Chlorophyll content is obtained by using the portable Chlorophyll Meter SPAD502 (Minolta Corporation, New Jersey, USA). In every measured paddy field, twenty rice samples are randomly selected, and then the averaged SPAD value of all leaves 
of each rice samples is viewed as chlorophyll content of the measured rice samples. Finally, the SPAD values of the twenty rice samples are further averaged as the rice chlorophyll content for the measured paddy field.

\subsection{Vegetation Indices Used in the Study}

In the study, the five vegetation indices, NDVI, MSR, MCARI, TCARI, and OSAVI are comparatively applied to assess the rice chlorophyll content.

NDVI (Normalized Difference Vegetation Index) should be the most extensively used VI which couples the maximum reflection in the infrared with the maximum absorption in the red, and is formulated with the following equation when using hyperspectral wavebands, where is $\mathrm{R}$ the reflectance at the given waveband $(\mathrm{nm})$ :

$$
N D V I[670,800]=\frac{R_{800}-R_{670}}{R_{800}+R_{670}}
$$

MSR (Modified Simple Ratio index) is the improvement on SR (Simple Ratio index). In hyperspectral remote sensing applications, SR is directly formulated with the reflection extremum in the infrared and that of the absorption in the red, and can enhance the contrast between soil and vegetation while minimizing the effect of the illumination conditions (Barnet and Guyot, 1991), but their effectiveness is reduced by the soil reflectance underneath the canopy. So MSR is proposed to further decrease the soil noise, and its quantified equation is as the following:

$$
\operatorname{MSR}[670,800]=\frac{R_{800} / R_{670}-1}{\sqrt{R_{800} / R_{670}}+1}
$$

Based on CARI (Chlorophyll Absorption Ratio Index) which measures the depth of chlorophyll absorption at $670 \mathrm{~nm}$ relative to the green reflectance peak at $550 \mathrm{~nm}$ and the reflectance at $700 \mathrm{~nm}$ in order to reduce the effect of some non-photosynthetic materials, MCARI (Modified Chlorophyll Absorption Ratio Index) is developed to further weaken the noise due to non-photosynthetic materials, as the following equation:

$$
\operatorname{MCARI}[670,700]=\left[\left(R_{700}-R_{670}\right)-0.2\left(R_{700}-R_{550}\right)\right]\left(R_{700} / R_{670}\right)
$$

TCARI (Transformed Chlorophyll Absorption Ratio Index) is proposed to eliminate reflectance effect of background matters (soil and non-photosynthetic components) and to increase the sensitivity at low chlorophyll content, and is formulated with the following equation:

$$
\operatorname{TCARI}[670,700]=3\left[\left(R_{700}-R_{670}\right)-0.2\left(R_{700}-R_{550}\right)\left(R_{700} / R_{670}\right)\right]
$$

OSAVI (Optimized Soil-Adjusted Vegetation Index) belongs to SAVI family (Huete, 1988), and is developed to further reduce background soils, and defined as the following:

$$
\operatorname{OSAVI}[670,800]=\frac{(1+0.16)\left(R_{800}-R_{670}\right)}{\left(R_{800}+R_{670}+0.16\right)}
$$


Vegetation spectrum from $400 \mathrm{~nm}$ to $700 \mathrm{~nm}$ region is primarily determined by chlorophyll and other pigments. The bands in the above five VIs is commonly selected to assess the chlorophyll content. In recent years, it is proved that besides the combination of the above bands, the new combinations of the other bands is possibly more sensitive and effective to leaf chlorophyll. For example, Sims and Gamon (2002) found that the band of $705 \mathrm{~nm}$ has better correlation with leaf total chlorophylls besides $680 \mathrm{~nm}$, and they also found a strong correlation between different combinations of reflectance at $700 \mathrm{~nm}, 705 \mathrm{~nm}$, and $750 \mathrm{~nm}$, and leaf chlorophyll content. Wu et al (2008) utilized the reflectance at $750 \mathrm{~nm}$ and $705 \mathrm{~nm}$ to form the revised VIs to estimate the chlorophyll of wheat and corn. Therefore, the reflectance of these bands may have potentials in assessing chlorophyll content. In this study, the above five VIs are modified to assess the rice chlorophyll content with the new combination of the reflectance at $750 \mathrm{~nm}$ and $705 \mathrm{~nm}$ replacing that of $800 \mathrm{~nm}$ and 670 $\mathrm{nm}$. The formulas of the five modified VIs are as Table 1.

Table 1. The formulas of the five modified VIs

\begin{tabular}{|c|c|}
\hline VI & Formula \\
\hline NDVI & $N D V I[705,750]=\frac{R_{750}-R_{705}}{R_{750}+R_{705}}$ \\
\hline MSR & $M S R[705,750]=\frac{R_{750} / R_{705}-1}{\sqrt{R_{750} / R_{705}}+1}$ \\
\hline MCARI & $\operatorname{MCARI}[705,750]=\left[\left(R_{750}-R_{705}\right)-0.2\left(R_{750}-R_{550}\right)\right]\left(R_{550} / R_{705}\right)$ \\
\hline TCARI & $\operatorname{TCARI}[705,750]=3\left[\left(R_{750}-R_{705}\right)-0.2\left(R_{750}-R_{550}\right)\left(R_{750} / R_{705}\right)\right]$ \\
\hline OSAVI & OSAVI $[7=705,750]=\frac{(1+0.16)\left(R_{750}-R_{705}\right)}{\left(R_{750}+R_{705}+0.16\right)}$ \\
\hline
\end{tabular}

\subsection{Weight Optimization Combination Method}

Weight optimization combination (WOC) is a method which calculates optimal weights of different models solving the same problem to form the combination model with the aim of the least errors (Xu et al., 2009). The principle of WOC is as the following:

There are different $N$ models constructed with $n$ samples for settling the same problem, where both $N$ and $n$ are the same as the following mentioned, respectively.

$y_{j}$ : the measured value for $j$ sample $(j=1,2,3, \cdots, n)$;

$f_{i j}$ : the estimated result for $j$ sample with $i \operatorname{model}(i=1,2,3, \cdots, N)$;

$e_{i j}=y_{j}-f_{i j}:$ the error for $j$ sample with $i$ model;

The estimated value of the combination model formed by $N$ models for $j$ sample is defined as the following:

$$
f_{j}=\sum_{i=1}^{N} k_{i} f_{i j}
$$


Here, $k_{i}$ are weights of $N$ single models, and $k$ is constrained by the following conditions:

$$
\left\{\begin{array}{c}
k_{i} \geq 0 \\
\sum k_{i}=1
\end{array}\right.
$$

The error of the combination model for $j$ sample is formulated with the following:

$$
e_{j}=y_{j}-f_{j}=\sum_{i=1}^{N} e_{i j} k_{i}
$$

For determining $k_{i}, e_{j}$ is usually looked on as independent variable of the objective function, and the mathematic framework of WOC is commonly expressed as the following:

$$
\left\{\begin{array}{c}
E=\min E\left(k_{1}, k_{2}, \ldots, k_{i}\right) \\
\sum k_{i}=1 \\
k_{i} \geq 0
\end{array}\right.
$$

where $\min E$ is the objective function that can be the minimum error square sum, or minimum absolute error sum, or the other cost function.

The process solving the formula (4) for the acquisition of $k_{i}$ is a little complicated, and there are various solving algorithms for the formula (4) with different effectiveness. In the study, the iterative optimization algorithm based on dual optimal combination is selected to calculate the optimal weights, whose detailed description can be found in Tang et al (Tang et al., 1994)

\section{Result and Discussion}

To analyze the performance of the five modified VIs for assessing chlorophyll content, the five VIs, respectively using the combination of $670 \mathrm{~nm} \& 800 \mathrm{~nm}, 705 \mathrm{~nm} \&$ $750 \mathrm{~nm}$, are correlated with the measured rice chlorophyll in 38 paddy fields from the two farms. Their correlation coefficients are as shown in Table 2.

Table 2. The correlation coefficients between the five VIs and SAPD chlorophyll content

\begin{tabular}{ccc}
\hline Vegetation index & R[670,800] & R[705,750] \\
\hline NDVI & 0.351 & 0.478 \\
MSR & 0.365 & 0.485 \\
MCARI & -0.345 & 0.453 \\
TCARI & -0.457 & -0.474 \\
OSAVI & 0.342 & 0.470 \\
\hline
\end{tabular}

From Table 2, we can see that after using the combination of $705 \mathrm{~nm}$ and $750 \mathrm{~nm}$, the correlations between the modified VIs and rice chlorophyll have more obvious increase in comparison with those of the initial VIs with $670 \mathrm{~nm}$ and 800nm. Although the correlation coefficient of TCARI with rice chlorophyll improves unconspicuously, 
the correlations of the other four VIs, NDVI, MSR, MCARI and OSAVI have been greatly raised.

In order to further compare the ability of the five modified VIs assessing rice chlorophyll, WOC is adopted by using the following steps: Firstly, the five models which correlate the five revised VIs with rice chlorophyll respectively are set up, the LLST(Linear Least Squares Fit) is the main modeling method in the study; Secondly, the five models viewed separately as the single model are input into WOC; Finally, the iterative optimization algorithm above mentioned is applied to calculate the weights.

In the process of using WOC with the iterative optimization algorithm, the more useful information the single model contributes to, the bigger weight it is given, and vice versa. That is to say, WOC has the function of judging redundant information (Tang, 1992). As far as the single model providing little or useless information is concerned, WOC would give the model less or zero weight. Therefore, in the study, we can determine which of the VIs are more probably sensitive to rice chlorophyll according to the weight when the five models constructed separately by the five modified VIs are input into WOC.

Table 3. Weights of the single model in the combining model based on WOC

\begin{tabular}{ccc}
\hline Model with different VI[705,750] & Weight & Least Sum of Square Error \\
\hline NDVI & 0 & 272.08 \\
MSR & 0.33 & 262.43 \\
MCARI & 0 & 273.65 \\
TCARI & 0 & 266.88 \\
OSAVI & 0.67 & 261.79 \\
Combination Model & -- & 261.60 \\
\hline
\end{tabular}

Table 3 shows the weights of the five models with the modified VIs based on WOC. From Table 3, the VIs MSR and OSAVI acquire non-zero weight, 0.33 and 0.67 respectively, and the other three are given zero weight, which illustrates that it would be enough to use the two VIs MSR and OSAVI when preparing to adopt the modified VIs at the same time to assess the rice chlorophyll. Moreover, Table 3 also tells us that the combination model has higher precision than each of the single models due to the minimum least Sum of Square Error.

\section{Conclusion}

In the study, the five typical vegetation indices, NDVI, MSR, MCARI, TCARI and OSAVI with usually using the combination of $670 \mathrm{~nm}$ and $800 \mathrm{~nm}$ are modified by replacing the two bands with $705 \mathrm{~nm}$ and $750 \mathrm{~nm}$ to assess rice chlorophyll content, and there are conclusions as the followings: 
(1) The five modified vegetation indices with the combination of $705 \mathrm{~nm}$ and $750 \mathrm{~nm}$ have better correlation with rice chlorophyll content in comparison in the five VIs with $670 \mathrm{~nm}$ and $800 \mathrm{~nm}$, which shows that the former has the potentials in assessing chlorophyll content.

(2) When using WOC to further compare the performance of the five modified vegetation indices for assessing rice chlorophyll, the two vegetation indices MSR and OSAVI show better performance than the other three VIs

The study only explores the ability of the five modified vegetation indices assessing rice chlorophyll for one rice growth period, and the following research work would focus on multi-periods.

Acknowledgement. Funding for this research is supported by 863 National High Technology R\&D Program (code: 2006AA210108, 2006AA10A302 \& 2006AA10A307) (P. R. China).

\section{References}

1. Niinemets, U., Tenhunen, J.D.: A model separating leaf structural and physiological effects on carbon gain along light gradients for the shade-tolerant species Acer saccharum. Plant Cell Environ. 20, 845-866 (1997)

2. Penuelas, J., Filella, I.: Visible and near-infrared reflectance techniques for diagnosing plant physiological status. Trends in Plant Science 3, 151-156 (1998)

3. Rasmus, H., Martha, A., Craig, D.: Utility of an image-based canopy reflectance modeling tool for remote estimation of LAI and leaf chlorophyll content at the field scale. Remote Sens. Environ. 113, 259-274 (2009)

4. Zarco-Tejada, P.J., Miller, J.R., Morales, A., Berjon, A., Aguera, J.: Hyperspectral indices and model simulation for chlorophyll estimation in open-canopy tree crops. Remote Sens. Environ. 90, 463-476 (2004)

5. Yongqin, Z., Jingming, C., John, R.M., Thomas, L.N.: Leaf chlorophyll content retrieval from airborne hyperspectral remote sensing imagery. Remote Sens. Environ. 112, 3234-3247 (2008)

6. Demarez, V., Gastellu-Etchegorry, J.P.: A modeling approach for studying forest chlorophyll content. Remote Sens. Environ. 71, 226-238 (2000)

7. Rasmus, H., Eva, B.: Mapping leaf chlorophyll and leaf area index using inverse and forward canopy reflectance modeling and SPOT reflectance data. Remote Sens. Environ. 112, 186-202 (2008)

8. Rouse, J.W., Haas, R.H., Schell, J.A., Deering, D.W., Harlan, J.C.: Monitoring the vernal advancements and retrogradation of natural vegetation. In: NASA/GSFC, Final Report, Greenbelt, MD, USA, pp: 1-137 (1974)

9. Gitelson, A.A., Merzlyak, M.N.: Spectral reflectance changes associated with autumn senescence of Aesculus hippocastanum L and Acer platanoides L leaves-spectral features and relation to chlorophyll estimation. J. Plant Physiol. 143, 286-292 (1994)

10. Chen, J.: Evaluation of vegetation indices and modified simple ratio for boreal applications. Can. J. Remote Sens. 22, 229-242 (1996)

11. Daughtry, C.S.T., Walthall, C.L., Kim, M.S., Brown de Colstoun, E., McMurtrey III, J.E.: Estimating corn leaf chlorophyll concentration from leaf and canopy reflectance. Remote Sens. Environ. 74, 229-239 (2000) 
12. Rondeaux, G., Steven, M., Baret, F.: Optimization of soiladjusted vegetation indices. Remote Sens. Environ. 55, 95-107 (1996)

13. Sims, D.A., Gamon, J.A.: Relationships between leaf pigment content and spectral reflectance across a wide range of species, leaf structures and developmental stages. Remote Sens. Environ. 81, 337-354 (2002)

14. Baret, F., Guyot, G.: Potentials and limits of vegetation indices for LAI and APAR assessment. Remote Sens Environ. 35, 161-173 (1991)

15. Huete, A.R.: A soil-adjusted vegetation index (SAVI). Remote Sens. Environ. 25, 295-309 (1988)

16. Chaoyang, W., Zheng, N., Quan, T., Wenjiang, H.: Estimating chlorophyll content from hyperspectral vegetation indices: Modeling and validation. Agricultural and Forest Meteorology 148, 1230-1241 (2008)

17. Xingang, X., Jihua, W., Wenjiang, H., Cunjun, L., Xiaodong, Y., Xiaohe, G.: Estimation of crop yield based on weight optimization combination and multi-temporal remote sensing data. Transactions of the CSAE 25(9), 137-142 (2009) (in Chinese with English abstract)

18. Xiaowo, T., Yong, Z., Changxiu, C.: An iterative algorithm for optimal combination forecasting of non-negative weights. Systems Engineering Theory Methodology Application 3(4), 48-52 (1994) (in Chinese with English abstract)

19. Xiaowo, T.: Study of combination forecasting error information matrix. Journal of UEST of China 21(4), 448-454 (1992) (in Chinese with English abstract) 